\title{
The Yan A. Su Laboratory
}

\section{Department of Biochemistry and Molecular Biology, Catherine Birch McCormick Genomics Center, The George Washington University School of Medicine and Health Sciences, Washington, DC, USA}

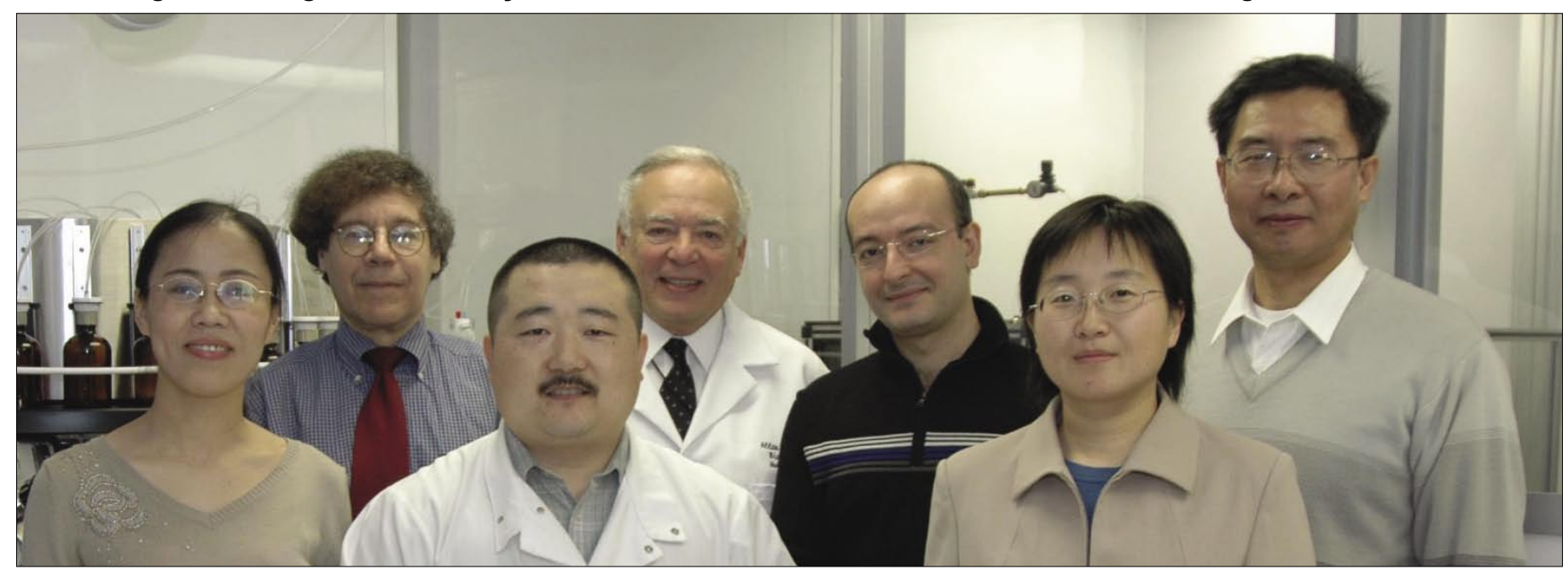

The People: From left to right: Qiuyang Zhang (Research Associate), Marc R. Blackman (Chief, NIH), Jun Wu (Research Associate), Allan L. Goldstein (Professor and Chair), Salvatore Alesci (Staff Scientist, NIH), Xueyan Bai (Research Associate), and Yan A. Su (Associate Professor and Associate Director).

\section{The Research}

In collaboration with our colleagues at the National Institute of Child Health and Human Development and the National Center for Complementary and Alternative Medicine at the $\mathrm{NIH}$, our laboratory has pioneered the development of novel human mitochondria-focused cDNA microarrays. These microarrays include hMitChip1 (first generation), hMitChip2 (second generation), and hMitChip3 (third generation) and customized bioinformatic tools for profiling gene expression focused on studying mitochondrial function in apoptosis and metabolic diseases. One of our research projects is to study the relationship between gene expression changes and cellular apoptosis of human malignant melanomas induced at multiple time points by UV irradiation, a major etiologic factor of skin cancer. Specifically, mitochondriarelated gene expression profiles of melanoma cells were compared with those of normal melanocytes at five different time points after UV irradiation. Another project involves the use of cancer cell lines and the hMitChip3 tools to investigate molecular mechanisms of the Warburg effect (i.e., switching of ATP-production from the oxidative phosphorylation pathway to the glycolytic pathway in malignant cells). Our results have revealed significant alterations in expression profiles of apoptosisand metabolism-related genes in melanoma cells in contrast to melanocytes. We are currently performing validation experiments for the expression profiles of resultant candidate genes.

\section{The Technique}

Our laboratory has developed DNA, antibody, and mutation microarrays and related bioinformatics tools (databases, computing procedures, and algorithms) for the study of coding and noncoding RNA expression, protein expression, DNA methylation, DNA-protein interactions, and gene mutations. In total, 19 unique types of microarrays, 12 databases, 1 algorithm, and 7 new software applications have been developed as highthroughput tools to facilitate both basic and applied biomedical research, maximizing the potential for discovery of new information from microarray datasets. With these technological tools, we emphasize not only a microarray experimental design, but we also provide investigators with novel integrative tools (i.e., gene chips together with databases and computing software). In addition, the microarray database of our gene chips are updated continuously using the most recent Human Unigene Database, downloadable from the National Center for Biotechnology Information (www.ncbi.nlm. nih.gov). By linking to public databases, it has allowed our investigators to instantaneously update the gene information at the time of data analysis.

Third-generation human mitochondria-focused cDNA microarray and its bioinformatic tools for analysis of gene expression, p. 365.

\section{www.gwumc.edu/biochem/faculty/su.html}

